\title{
BMJ Open Assessing the quality of medication documentation: development and feasibility of the MediDocQ instrument for retrospective chart review in the hospital setting
}

Antje Hammer (D) , ${ }^{1}$ Anke Wagner, ${ }^{2}$ Monika A Rieger, ${ }^{2}$ Tanja Manser, ${ }^{3}$ on behalf of the WorkSafeMed Project Consortium\#

To cite: Hammer A, Wagner A, Rieger MA, et al. Assessing the quality of medication documentation: development and feasibility of the MediDocQ instrument for retrospective chart review in the hospital setting. BMJ Open 2019;9:e034609. doi:10.1136/ bmjopen-2019-034609

\section{- Prepublication history for} this paper is available online. To view these files, please visit the journal online (http://dx.doi. org/10.1136/bmjopen-2019034609).

Received 27 September 2019 Revised 23 October 2019 Accepted 23 October 2019

Check for updates

(C) Author(s) (or their employer(s)) 2019. Re-use permitted under CC BY-NC. No commercial re-use. See rights and permissions. Published by BMJ.

${ }^{1}$ Institute for Patient Safety, University Hospital Bonn, Bonn, Germany

${ }^{2}$ Institute of Occupational and Social Medicine and Health Services Research, University Hospital of Tübingen, Tuebingen, Germany

${ }^{3}$ School of Applied Psychology, University of Applied Sciences and Arts Northwestern

Switzerland, Olten, Switzerland

Correspondence to

Dr Antje Hammer;

Antje.Hammer@ukbonn.de

\section{ABSTRACT}

Objective The medication process requires clear and transparent documentation in patient records. Incomplete or incorrect medication documentation may contribute to inappropriate clinical decision-making and adverse events. To comprehensively assess the quality of inhospital medication documentation, we developed a retrospective chart review (RCR) instrument. We report on the development process, the feasibility of the instrument and describe our application of the instrument to a sample of patient records.

Design Cross-sectional study using an RCR instrument to evaluate paper-based, non-standardised prescription and medication administration charts (MediDocQ).

Setting Two German university hospitals.

Participants Records from 1361 patients admitted between April and July 2015 were evaluated.

Methods The MediDocQ development process comprised six consecutive stages: focused literature review, webbased search, initial patient record screening, review by project advisory board, focus groups with professionals and pilot testing. The final 54-item RCR instrument covers three key components of medication documentation: (1) completeness of documented information (including prescription, medication administration and pro re nata (PRN) medication), (2) quality of transcriptions and (3) compliance with chart structure, legibility, handling of deletions and chart corrections. Descriptive statistics are presented as mean values, SD, median and interquartile ranges for individual items.

Results Overall, 33 out of 54 items resulted in mean values above 0.75 , indicating high-quality medication documentation. Documentation quality was particularly compromised for verbal and PRN orders (which involve more steps than standard orders) and when documentation was not completed at the same time as medication administration.

Conclusions MediDocQ is a patient safety instrument that can be used to evaluate the quality of medication documentation and identify components of the process where intervention is required. In our setting, standardisation of medication documentation, particularly regarding medication administration and PRN medication is a priority.

\section{Strengths and limitations}

- MediDocQ was developed based on a structured six-stage process (ie, focused literature review, web-based search, initial patient record screening, review by project advisory board, focus group review with healthcare professionals and pilot testing).

- In developing MediDocQ, we addressed the interprofessional nature of the medication process by including patient record entries by both physicians and nurses to assess medication documentation quality throughout the continuum of care in hospitalised patients.

- By focusing on the quality of medication documentation, MediDocQ neither aims to detect actual medication errors nor check for the correct indication of the prescribed medication.

- As with all retrospective chart reviews, the MediDocQ findings depend on the accuracy of the information presented in the available documentation.

\section{INTRODUCTION}

Delivering high-quality patient care is highly dependent on the accurate, reliable and timely flow of information. However, effective information transmission along the continuum of care is hindered by poorly designed work processes, frequent handover and transitions, and the multiprofessional nature of complex patient care processes. ${ }^{1-6}$ The quality of documentation in patient records plays a key role in supporting the optimal flow of care-related information as it complements verbal communication by capturing clinical assessments, treatment decisions and actions taken by different care providers along the continuum of care. Thus, patient records provide an essential reference for care providers' decision-making and there is a legal requirement in most healthcare 


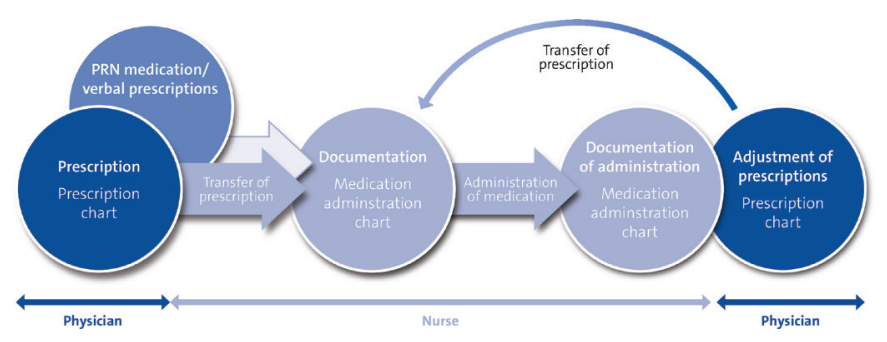

Figure 1 Work and information flow in the medication process adapted from Hartel et $\mathrm{al}^{14}$. PRN, pro re nata.

systems to document all patient-relevant information ${ }^{78}$ and decisions during a hospital stay.

One crucial component of medical treatment is medication. Medication-related adverse events (MRAE) are among the most common healthcare-related adverse events. ${ }^{9-12}$ The medication process ${ }^{13}$ frequently involves several interfaces between different care professionals within or across hospital units, necessitating clear and transparent documentation. Prescription orders and medication administration documented in patient records allow nurses and physicians, working across time and locations, to access relevant information at any point in time. ${ }^{7}$ Moreover, the medication process comprises several error-prone steps that require not only high levels of staff concentration (eg, preparing medications) but also timely access to information so that staff is fully briefed about patient care (eg, new orders, verbal prescriptions and reasons for ceasing medication).

The in-hospital medication process (figure 1) in Germany includes several activities performed by either nurses or physicians: physicians prescribe medications on prescription sheets (physicians' responsibility) and nursing staff transcribe these orders onto medication administration charts (nurses' responsibility). Both charts are part of the patient record and accessible by all professionals involved in patient treatment. In the course of treatment, nurses primarily administer medications and document the administration in the medication administration chart (nurses' responsibility and rarely physicians' responsibility). Any prescription adjustments (including ceasing medications) are made by the treating physician on the prescription chart (physicians' responsibility). The transcription and documentation of adjustments are again performed by the nurse on the medication administration chart (nurses' responsibility). ${ }^{14}$ In most German hospitals, pharmacists are available on request but are rarely directly involved in this process.

To reduce the risk of MRAEs caused for example by transcription errors, several countries such as Australia, the UK and the USA, have established explicit standards for medication documentation throughout the medication use process. The majority of their hospitals now use tools such as a national inpatient medication chart (that combines prescription and administration documentation into a single chart $)^{15} 16$ or electronic Medication Management Systems. ${ }^{17}$ Nevertheless, many countries, including Germany, still lack standardisation of documentation practices and are faced with local documentation practices that differ not only between hospitals, but also between units. This lack of standardisation can impair information transfer and cause MRAEs.

Retrospective chart review (RCR), also known as medical record review or patient record review, is a common data collection approach that involves the extraction and evaluation of existing patient data from patient records ${ }^{18}$ using a standardised set of questions or probes. Measuring patient safety with RCR data is an established approach for obtaining objective data on patient safety at the patient level and to identify areas for improvement related to structures and processes at a unit level or hospital-wide. ${ }^{19}{ }^{20}$ In comparison to other data, such as routinely collected administrative data or critical incident reports, RCR data enable an accurate, direct and quantitative analysis of patient care data. ${ }^{21-24}$

Several studies exist to measure MRAE in healthcare. $^{25-27}$ These studies either use different observational study designs (eg, clinical audits or surveys) [eg, refs ${ }^{28-31}$ ], refer to medication processes at hospital admission or discharge [eg, refs ${ }^{32-34}$ ] or focus on the prevalence of a specific MRAE (eg, overdose or contraindication) [eg, refs 20252635 . Few studies also assessed the quality of medication documentation but focused either on the completeness of record components (eg, physician orders, nursing notes, diagnostic results, medication list and discharge letter) and the availability of certain patient information (eg, reasons for admission) ${ }^{36}$ or the documentation in certain specialities such as surgery. ${ }^{14}$ However, within this study, we sought to assess the quality of nurses' and physicians' medication documentation and to reflect the documentation of the in-hospital medication use process that may contribute to certain adverse events. Therefore, we developed an RCR instrument specifically designed to assess the quality of medication documentation (MediDocQ) in the in-hospital medication process. We report the instrument development process and demonstrate its feasibility in the hospital setting. Based on descriptive findings from two German university hospitals, we describe our application of MediDocQ to a sample of patient records.

\section{METHODS}

\section{Study context and population studied}

The instrument development process described here was part of the larger research project 'Working conditions, safety culture and patient safety in hospitals-what predicts the safety of the medication process?' (WorkSafeMed) that examined the associations between frontline staff perceptions of working conditions, patient safety, occupational safety climate and patient safety outcomes. WorkSafeMed was a cross-sectional study conducted between 2014 and 2017 in two German university hospitals. ${ }^{37}$

In order to obtain sufficient analysable patient records per unit for the RCR, we included inpatient units treating at least 500 patients per year from the two participating 


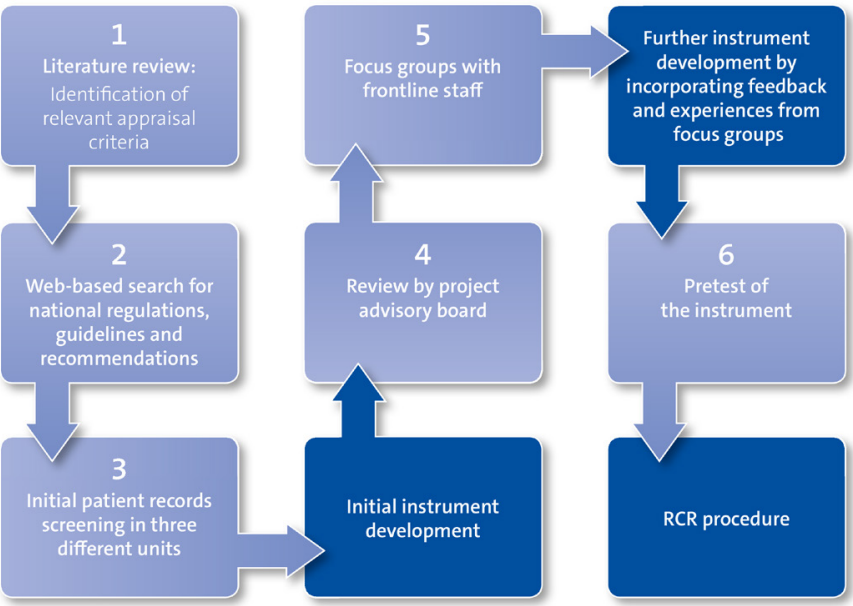

Figure 2 Six stages of the RCR development process and testing. RCR, retrospective chart review.

university hospitals. We excluded specific units such as intensive care and psychiatric units because their patient record documentation differs or is often more complex and is therefore not comparable with the documentation from other units. In total, 73 units from 37 departments were eligible for inclusion in the WorkSafeMed study. Out of these, 65 units from 29 departments agreed to participate in the RCR. At the time of the study, none of the units used a unit-dose-drug-distribution system (UDDS) ${ }^{38}$ or an electronic documentation system.

We sought to review a purposeful sample of 25 patient records per unit, a total of 1625 patient records, from patients admitted between April and July 2015. For informed consent, we approached individual units, inviting all patients present in the unit at that time. Patients with cognitive disorders (eg, confusion or dementia), speaking neither German nor English, or underage children without a guardian available, were excluded. Units were approached several times until at least 25 patients agreed to participate. Where possible, we oversampled by five patients.

\section{Patient and public involvement}

Patients and the public were not involved in the instrument development, analysis or interpretation of the data. However, we thank all patients, who agreed to data collection from their charts for this study.

\section{Development of MediDocQ}

The development and testing of the MediDocQ instrument included six consecutive stages (figure 2). First, we conducted a focused literature review using PubMed and Web of Science to identify relevant appraisal criteria for evaluating the quality of medication documentation in hospitals by RCR. Search terms used were 'documentation', 'patient chart', 'patient record' and 'medication'. This literature review was complemented in the second stage by a web-based search for international and national regulations, guidelines and recommendations concerning medication documentation in patient records. Based on repetitively mentioned and legally required aspects, we identified five core requirements for medication documentation: document authenticity, transparency, clarity, completeness and timeliness (table 1). ${ }^{7839-41}$

Then, seeking to develop appraisal criteria applicable across hospital departments and units with different local medication documentation practices we screened patient records in three different units (dermatology, surgery and ear, nose and throat units). Units were chosen to represent a wide range of medication and routes of their administrations (eg, oral, rectal, topical, subcutaneous and intramuscular). Based on the first three stages, we drafted an initial version of the RCR instrument that considered the five core requirements for medication documentation in patient records. To ensure, for example, transparent and timely documentation and administration, medical prescriptions should be signed/initialled by the physician

Table 1 Core requirements for documentation

\section{Requirement Explanation}

Document The documentation must be legibly written using indelible writing materials such as black or blue ink, but not authenticity pencil, which can be easily erased from the record ${ }^{424445}$

Transparency To track treatment decisions and medication orders or the administration of medication, all entries should be dated, timed and signed/initialled to identify the nurse or physician responsible for the entry. Deletions or alterations of any entry must be trackable and should be dated, timed and signed off. ${ }^{74445}$ Correction fluid does not ensure the authenticity of patient documentation ${ }^{42} 45$

Clarity Documentation must be unambiguous. Symbols and abbreviations, especially if not commonly used might lead to misunderstandings. ${ }^{845}$ In the case of medications to be given immediately after prescription, additional information by the physician, such as 'give now' or 'give today at 10:00' might be helpful for the nurse to understand exactly when the treatment is expected to be given

Completeness All relevant information for the treatment of patient as well as decisions made during the patient stay, including verbal or telephone orders and prescriptions must be documented ${ }^{84245}$

Timeliness Record entries should include the date and time, and must be made in a timely manner (eg, medication administration should be documented immediately after medications are administered, verbal/telephone orders must be documented in a timely manner and signed by a physician as soon as possible) $)^{84244}$ 
and documented with the date and time (generic aspect GO1).

In stage 4, this version was reviewed and discussed in detail by the project advisory board, which comprised experts in the fields of clinical pharmacology (2), patient safety (1), nursing (1) and healthcare quality (1). After incorporating their suggestions, we sought feedback on the instrument in stage 5 by introducing it to frontline staff in two focus groups, one at each hospital. Ten nurses and seven physicians from different departments (ie, dermatology, internal medicine, general surgery, gynaecology, neurosurgery, ophthalmology, radio oncology and trauma surgery) participated in the focus groups. Participants were first asked about daily problems and general difficulties concerning medication documentation. Then, they were asked to describe indicators of high quality in the documentation of the medication process. In a final round, participants were introduced to the current draft of the instrument and asked to rate whether the instrument covered all relevant aspects of quality of medication documentation and whether the items were complete and their meaning was clear.

Feedback from focus groups was received regarding local practices in prescribing medication. For example, some units have nurse-initiated medication procedures which allow them to administer commonly used medications listed on an approved medication list without prescription by a physician. Local practices were also reported regarding the documentation of medication administration. For example, some units use a two-step procedure, first documenting the medication preparation with a pencil in the medication administration chart and then overwriting it with indelible ink once the medication has been administered. Other units tick off or initial every single medication when administered to the patient and other units simply tick off medication administration once a day, without initialling. We further developed the instrument by incorporating the focus group input and pilot-tested the new version in stage sixth with a total of 13 patient records from different departments. The pilottest resulted in minor changes in the wording of some items. No significant changes were required resulting in the MediDocQ development process being finalised.

Within the purpose of the WorkSafeMed study and for feasibility reasons, MediDocQ is not intended to identify actual medication errors (eg, overdose and drug interactions), nor does it assess the appropriateness of the indication for a medication.

\section{Content of the MediDocQ instrument}

The MediDocQ instrument covers three key components in medication documentation quality: (1) completeness of information (eg, in prescriptions and adjustments of medications; in documentation of medication given); (2) quality of transcription (eg, from prescription chart to medication administration chart); and (3) compliance with chart structure, legibility and handling of deletions and corrections in charts.
With a total of 54 items, MediDocQ is structured into 8 themes and 16 topics. MediDocQ as well as the data collection and analyses manual are available on request by contacting the first author. Eleven out of 16 topics (G01-G11) are generic and applicable to all patient records, whereas items of the additional 5 topics (A01A05) were only rated if applicable (eg, A1: 'pro re nata (PRN) medication' was only rated when it was prescribed and/or administered). Most items were rated on ordinal 4-point Likert scale, some items were dichotomous no/ yes answers or continuous counts. For items with ordinal Likert scales (1=no, 2=somewhat no, 3=somewhatyes, $4=y e s)$, reviewers were instructed to rate according to whether a given item was complete for; no medications, less than half, more than half or all medications (eg, item 1: prescriptions were assigned with date was rated as somewhat yes (3), when more than half of prescribed medications were prescribed with a date). Items with no/yes answers $(0=$ no, $1=$ yes $)$ were rated according to whether this given item was not true (0) or true (1) (eg, item 40: pencil documentation in the prescription chart was rated with yes (1) when reviewer found any information documented in pencil).

\section{RCR procedure}

A total of nine reviewers with either medical or pharmaceutical background and with clinical experience reviewed the patient records. Reviewers underwent a 1.5day reviewer training course. Training materials served as reviewer manuals and included specific instructions for each item, example pictures for ratings and hints on where to find relevant information in patient records. All reviewers were instructed to focus on certain charts of the patient records: (1) prescription chart for rating the quality of physicians prescriptions, (2) medication administration chart for rating the quality of the transcription of medical prescriptions into nurse documentation and the quality of documentation of medication administration, and (3) a pro re nata medication box, which is usually separated from general medication and requires different information (such as specified symptoms or indications for use and maximum daily dose). Reviewers were instructed to review additional charts such as medication plans or daily shift notes from nurses or physicians, if necessary (eg, in cases where medications were not documented as administered, to identify the underlying reason). As MediDocQ does not capture actual medication errors, we considered the review of at least one prescription sheet and a maximum of two medication administration sheets sufficient to rate the quality of medication documentation. Thus, for patients with a long hospital stay, the review was stopped at the transcription of medical prescriptions of the second medication administration chart that usually started on day 8 of the hospital stay.

To ensure familiarity with specific medications or handling of documentation at the unit level, the same reviewer screened all records from one unit. During 
data collection, reviewers were supervised by local study coordinators $(\mathrm{AH}, \mathrm{AW})$ who ensured timely and direct information exchange. Decisions concerning specific reviewer questions were documented and shared immediately with all reviewers. For quality assurance, $10 \%$ of the patient records were reviewed independently by a second reviewer. If necessary, discrepancies were resolved by consensus discussions between reviewers and the local study coordinator.

\section{Statistical analyses}

All records with at least one documented medication were included in the analyses presented. We did not exclude cases due to missing values in individual items. Reverse coded items were recoded and all items were normed to values between $0=$ poor and $1=$ excellent. For unique descriptive description and comparison, we report mean values and SD on the normed values of individual items. Values $\geq 0.75$ were considered as indicating high-quality medication documentation. Additionally, we calculated the median and interquartile ranges on the original answer scale for individual items.

\section{Ethics and consent to participate}

The WorkSafeMed study with all its components was approved by the responsible ethics committees of the medical faculties of the project partners in Bonn (\#350/14) and Tubingen (\#547/2014BO1). Each partner complied with confidentiality requirements according to German law. Prior to data collection, we gained informed consent from the patients involved in the chart review.

\section{RESULTS}

\section{Sample and feasibility}

We obtained access and informed consent for 1630 patient records. After excluding records due to missing prescription or medication administration charts, 1361 patient records were reviewed. A total of 149 patient records (10.9\%) were independently reviewed by a second reviewer for quality assurance. Discrepancies were mostly found in the rating of Likert-scales (eg, one reviewer rated $4=$ 'yes' on item 1 , the other $3=$ 'somewhat yes') and were resolved by consensus discussions between reviewers and the local study coordinator. The reviewed patient records were either original paperbased records or scanned records. The quality of patient records reviewed was sometimes suboptimal (eg, item 40 and 45; pencil documentation was illegible in the case of scanned patient records) and some records were missing the necessary charts for this review. Thus, some items included missing values.

Out of all patient records, 1291 included at least one documented medication and were included in our analysis. Depending on the number of prescribed medications, the average review time per patient record ranged between 30 and $45 \mathrm{~min}$. The general descriptive statistics of the patient records are summarised in table 2. The average number of prescribed medications at admission was $7.98(\mathrm{SD}=5.42 ;$ median=7). The average length of stay in our sample was 8.63 days ( $\mathrm{SD}=10.96$; median $=5$ days). Regarding the number of charts, we found that medical prescriptions were made in only one chart in 1026 (79.5\%) patient records. In most cases, this was either

Table 2 General descriptive statistics of the patient records used in the analysis $(n=1291)$

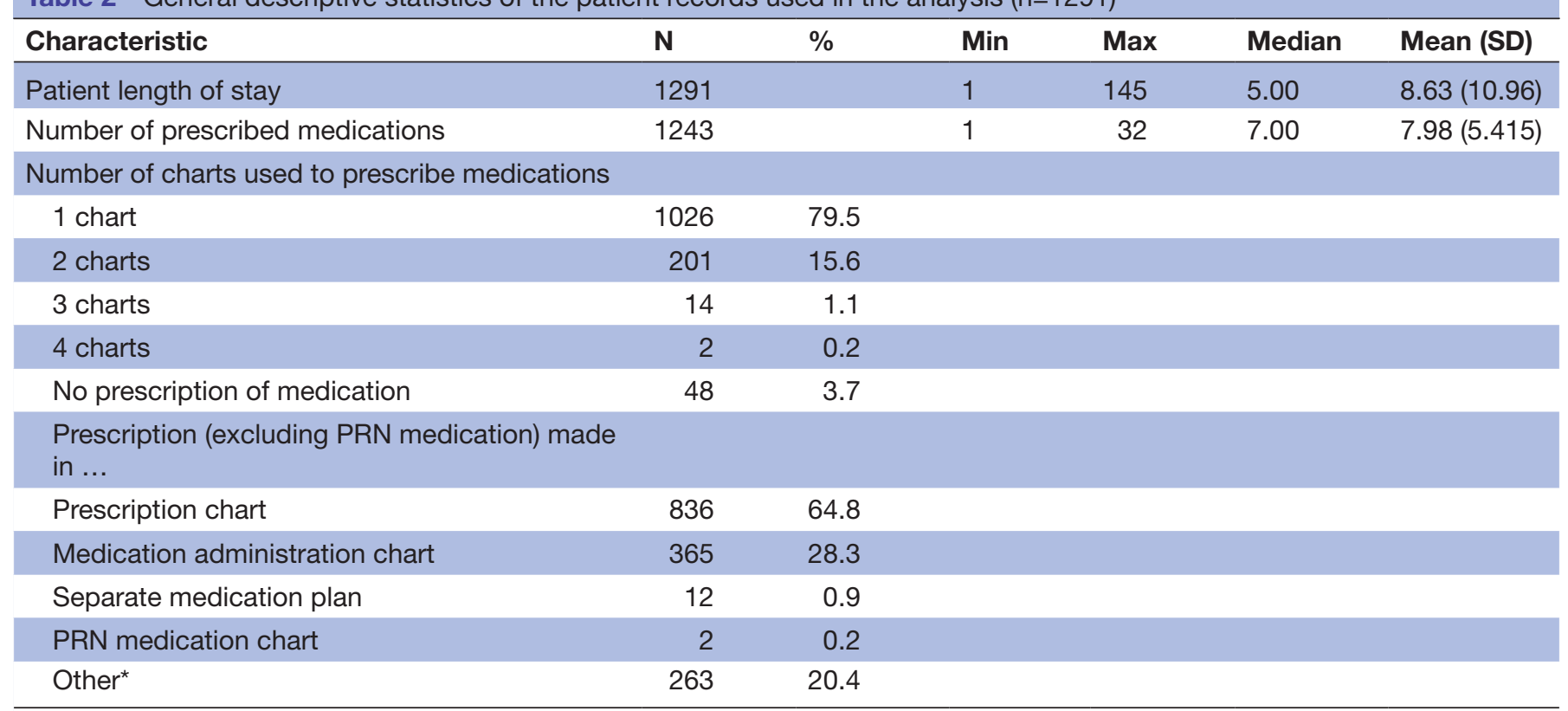

${ }^{*}$ Other chart information on prescriptions found were, for example protocols from emergency departments or anaesthesia protocols, discharge letter, additional medication plans such as chemotherapy or insulin plans.

PRN, pro re nata. 
the prescription chart or one of the medication administration charts, with medication prescribed directly by physicians. In 217 patient records, medical prescriptions were made in two or more charts (eg, in the prescription chart and medication plan or postoperative orders). In 48 patient records $(3.7 \%)$, reviewers were unable to find a medical prescription for medications documented in the medication administration chart.

Results for individual items are summarised in table 3. For an easier and unique descriptive comparison in the following, we refer to mean values and SD of normed items. Median and interquartile ranges on originally scaled items can be found in table 3 .

\section{Completeness of information}

Our results indicate a high quality of medication documentation with regard to 'duplicated medication prescription' made by physicians (G03: item 9, mean $=0.92$, $\mathrm{SD}=0.28)$ and 'completeness of medical prescriptions' (G02: for example, item 7, mean $=0.94, \mathrm{SD}=0.14$ ). Considering 'date, time and signature of medical prescriptions' (G01), most medical prescriptions were signed/initialled and dated, but only a few physicians stated the time when the medication was prescribed (item 2 , mean $=0.18$, $\mathrm{SD}=0.35$ ). One possible reason for this was the chart structure did not typically include a designated area for the time of prescription.

We found noticeably poor documentation quality for single items of 'PRN medication' (A01). Despite PRN medication boxes in medication administration charts usually providing enough space for specifying symptoms or indications for medication use, a large number of PRN prescriptions lacked this information (item 16, mean $=0.17, \mathrm{SD}=0.35$ ). Poorest documentation quality was identified for 'telephone/verbal prescriptions' (A02). This was found in only 134 patient records, mostly in the nursing documentation but was rarely countersigned by a physician (item 21, mean $=0.14, \mathrm{SD}=0.34$ ).

\section{Quality of transcription}

We found a high quality of documentation concerning the transcription of medical prescriptions to the first medication administration chart (G06). However, we identified discrepancies between the number of medications documented in the prescription chart and the medication administration chart (item 22, mean $=0.59, \mathrm{SD}=0.49$ ). Reviewers mentioned that the reasons for these discrepancies were the documentation of medication that was not prescribed or the transcription of medication directly from postoperative orders or intensive care unit documentation after patient transfer without documented prescription by the unit's physician. Also, the 'transcription of adjustments in medication' was of moderate documentation quality (G08: item 35 , mean $=0.68, \mathrm{SD}=0.42$ ). Similarly, the documentation quality for the administration of medications not prescribed was found to be poor (item 33, item mean $=0.46, \mathrm{SD}=0.50$ ). Furthermore, we found lower documentation quality with regard to the 'standards for documentation of medication administration' (G04: eg, item 10, mean=0.45, SD=0.41) and found a high number of missing documentation for 'medication administration' (G05: for example, item 14, mean $=0.57$, $\mathrm{SD}=0.50$ ). Results showed very high documentation quality for the transcription of medications from the first to second medication administration chart (A03: eg, item 30 , mean $=0.98, \mathrm{SD}=0.10)$ and the 'timely adjustment of medication' (G09: item 36, mean $=0.95, \mathrm{SD}=0.15$ ).

\section{Compliance with chart structure, legibility and handling of deletions and corrections}

We found high compliance with regard to chart structure and legibility in both the prescription chart (G10: eg, item 41 , mean $=0.93, \mathrm{SD}=0.26$ ) and medication administration chart (G11: eg, item 44 , mean $=0.89, \mathrm{SD}=0.31$ ). However, ratings on deletions/corrections resulted in relatively low values for both the prescription chart (A04: eg, item 49 , mean $=0.02, \mathrm{SD}=0.13$ ) and medication administration chart (A05: eg, item 52, mean $=0.02, \mathrm{SD}=0.13$ ). Only in a few cases of deletion, the original text was still legible. Almost all deletions were neither initialled nor dated.

\section{DISCUSSION}

In this study, we developed and tested MediDocQ, an RCR instrument for assessing the quality of medication documentation in the hospital setting. The instrument covers three key components: (1) completeness of information concerning prescription, documentation of medication administration, adjustment of medication, and documentation of verbally communicated and PRN medication; (2) quality of transcriptions (eg, from the prescription chart to the medication administration chart) and (3) compliance with chart structure, legibility, handling of deletions and corrections in charts.

\section{Feasibility when using MediDocQ}

During RCR development and data gathering, we became aware of a high degree of heterogeneity in the departmentspecific and unit-specific documentation practices. Moreover, the feasibility was compromised when the quality of the review material was low due to scanned or incomplete documents. To control for such local practices, reviewers were instructed to report such details in one general open-ended question (eg, PRN medication never documented in prescription charts; crosscheck with the unit for this being nurse-initiated medication). Moreover, all MediDocQ items represent different levels of importance. Although some items are essential ('must have') to ensure a safe medication process, such as complete information on dose, dose interval and route of administration, other items refer to information supporting transparency in the medication process if documented ('nice to have'). For example, stating the time of day on a prescription would be very helpful with regard to the timeliness and transparency of newly prescribed, altered or ceased medications. However, this information is not legally required and 


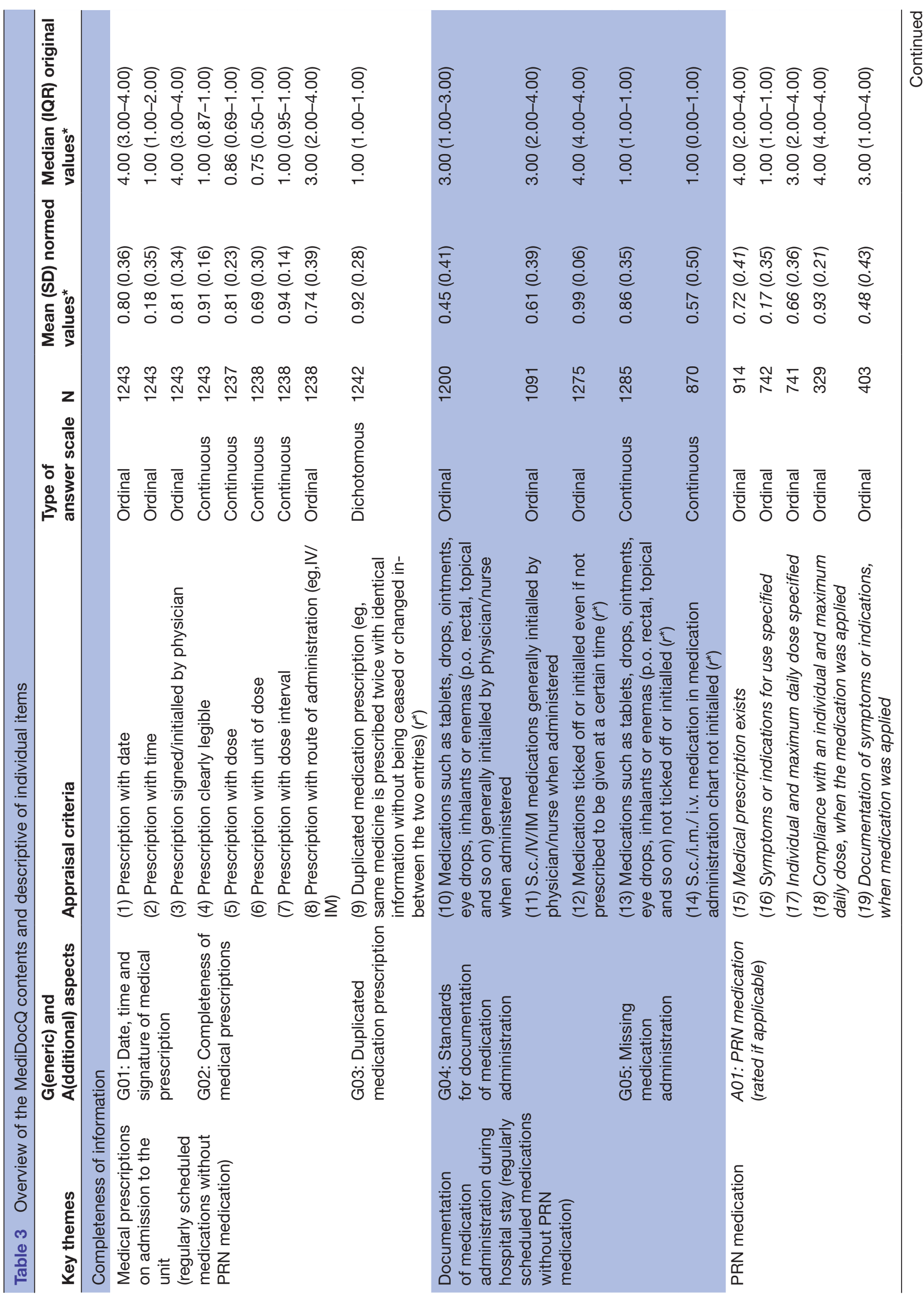




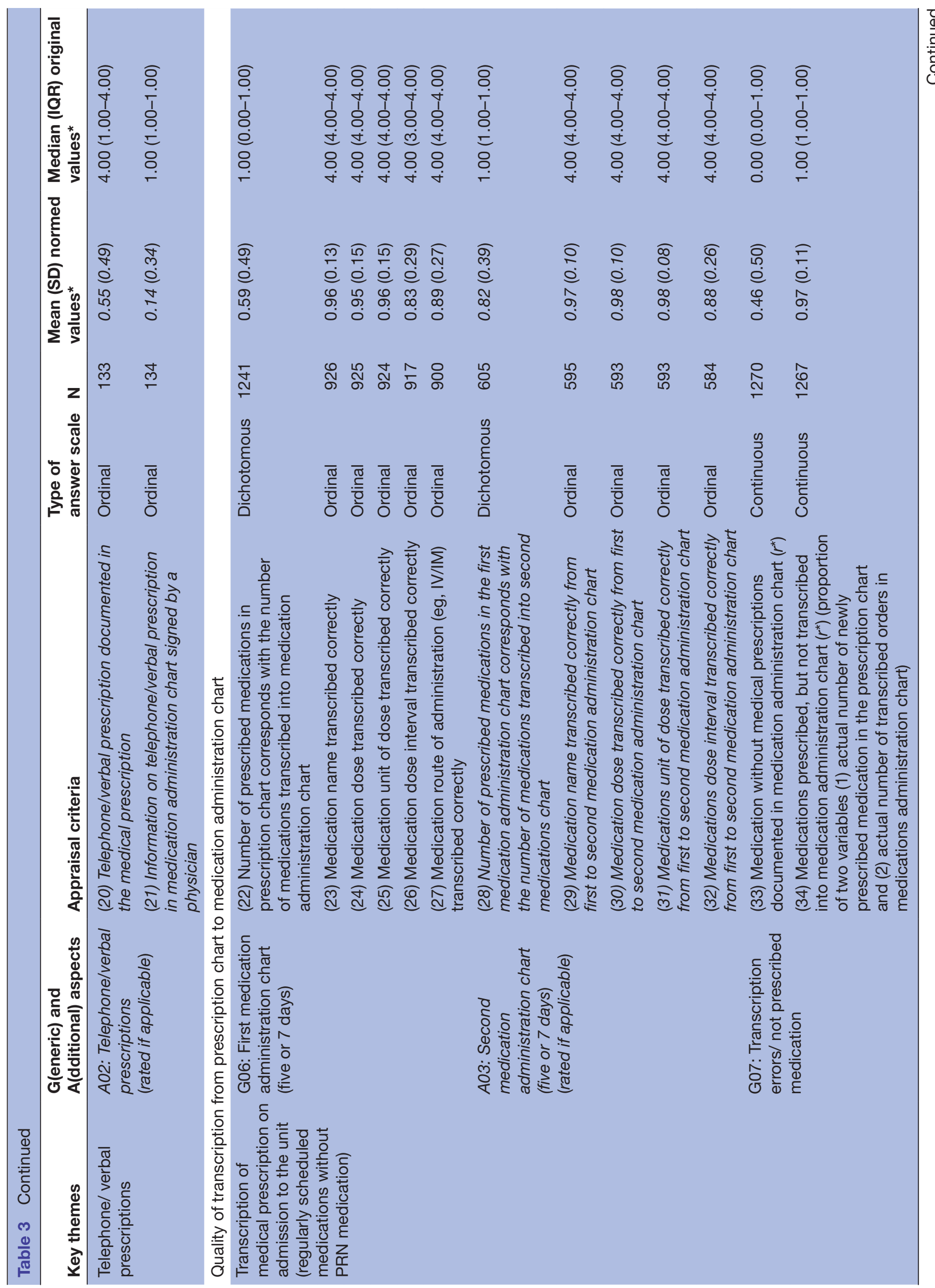




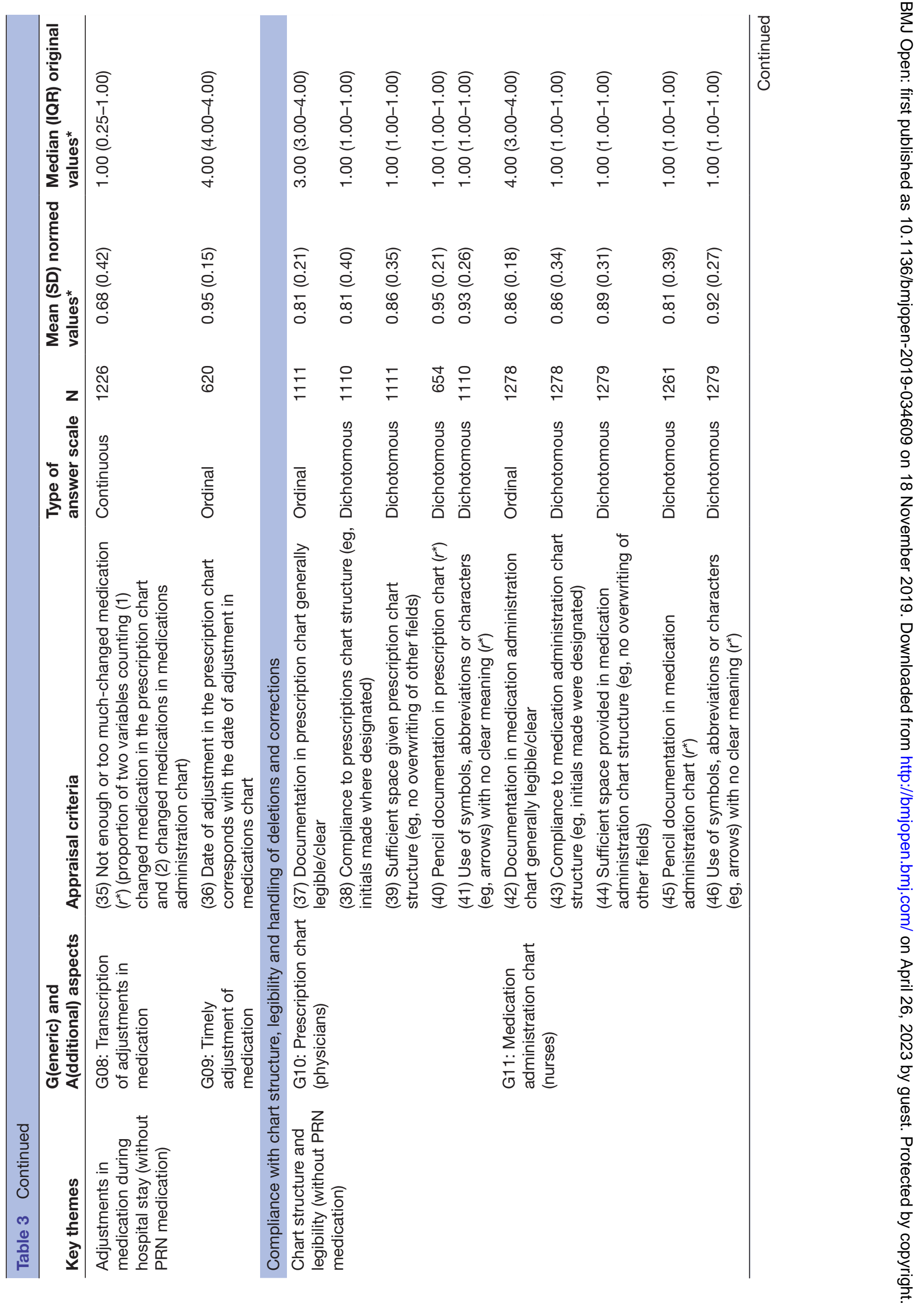


most prescription charts did not provide enough space for such information.

\section{Assessing the quality of documentation in the medication process}

Overall, our results show a positive, yet heterogeneous picture of documentation quality. In total, 33 of the 54 items reached values above 0.75 , indicating a high documentation quality in the medication use process. Although results were generally positive, documentation quality was particularly compromised when documentation was delayed (eg, documentation of verbal prescriptions that were not written in a timely manner and administration was delayed because the nurse was interrupted) or when additional effort was required to ensure documentation (eg, nurses may document medication administration at the end of their shift, instead of at the moment of administration, because patient records are needed in medical rounds). The review process revealed striking variations in local documentation practices and a lack of medication documentation standards across units and departments. Thus, we identified that standardisation of the documentation of medication administration is a key area for improvement. The lack of documentation of medication administration can lead to MRAE, such as medication overdose when medications are administered twice. Another area of improvement concerns the documentation of PRN medication, especially with regard to the documentation of symptoms/indications for medication use. In both cases, we identified a need for common standards in medication documentation.

\section{Strengths and limitations}

MediDocQ reflects the interprofessional nature of the medication process by assessing physicians' and nurses' medication documentation. The involvement of nurses and physicians from different units and departments in the RCR development process and the consideration of their experiences from daily work processes enabled the RCR instrument to address specific practices at the unit and department levels.

Nevertheless, this RCR instrument has some limitations with regard to its content and implementation. The MediDocQ content is designed to focus on the quality of medication documentation. It neither detects actual medication errors nor checks for the correct indication of the prescribed medication. In addition, due to its content, MediDocQ required the rating of single items on different types of answer scales. For uniform descriptions, we normed these different types of answer scales (dichotomous, ordinal, and continuous) to values between $0=$ poor and $1=$ excellent; however, interpretations of results must consider the different types of scales in the original answers.

Considering the number of reviewers in this study and the fact that each unit's patient records were assessed by only one reviewer, there is a potential for reviewer bias. To minimise reviewer biases, two reviewers independently 
assessed a sample of $10 \%$ of all reviewed records and discrepancies were discussed among reviewers with the local study coordinator. Moreover, a standardised reviewer-training programme, using training materials as manuals and continuous supervision by local study coordinators ensured a high level of consistency in the data.

Concerning potential sampling bias, certain patient groups (eg, patients with cognitive disorders or without an available guardian) are not represented in our study. Finally, the accuracy of results from RCR tools is reliant on the general content and quality of patient record documentation and the information available. For example, in this study, we found a variety of compositions of charts in patient records and structures of charts at the unit and department levels.

\section{Implications for research and practice}

The main practical implication of this study is the need to define clear requirements for medication documentation in standards and guidelines, which consider legal requirements but are flexible enough to allow for local tailoring. The medication documentation process investigated in this study is quite complex (eg, documentation in different charts and transcription of physician orders into medication administration charts instead of using one singlepatient medication chart). We therefore recommend the implementation of more efficient solutions to reduce the complexity of documentation processes by removing redundant or irrelevant documentation tasks and excessive documentation. This recommendation becomes even more relevant considering the growing introduction of electronic documentation in hospitals often leading to parallel use of electronic and paper-based documentation during implementation. ${ }^{39} 40$ However, a complete switch to electronic documentation does not necessarily imply higher quality in all aspects of medication documentation but will reveal new challenges. ${ }^{40}$ Compliance with chart structure, legibility and handling of deletions and corrections will be less important in electronic patient records and relevant issues such as the date, time and signature of medical prescriptions can be recorded automatically. Forcing functions can be included in these systems to maximise the completeness of medical prescriptions, task reminders to administer medications can be included. However, documentation deficiencies may still occur with electronic systems; examples include prescriptions for the wrong patients, medication selection errors and inadequate description of the reasons for missed doses. ${ }^{41}$

To improve medication documentation, documentation in general needs a different standing in healthcare processes. For example, in Germany, the documentation in patient records serves as important legal evidence when pursuing claims of patient harm. ${ }^{42}$ However, the relevance of documentation in clinical practice is still not appreciated. For many healthcare professionals, the significance of the documentation process is not entirely clear and has thus become a bureaucratic, necessary 'evil' rather than a form of communication with colleagues about the details of patient care. Moreover, the documentation process is frequently compromised by other priorities, and documentation is often afflicted by missing real-time documentation or too many interruptions during the documentation in everyday work-flow. ${ }^{43}$ These are highly relevant problems that will not be erased by implementing electronic documentation. Thus, we propose giving more priority to documentation processes in healthcare by implementing compulsory introductory training or standardised use of introductory programmes for new staff members. Moreover, in order to raise awareness for documentation as early as possible, the requirements of the documentation process should be included in the education of nurses and physicians.

\section{CONCLUSIONS}

MediDocQ can be used to evaluate the quality of the medication documentation process, which is an important aspect when aiming to understand and improve patient safety. The instrument reveals areas for interventions in medication documentation, where improvements are crucial. The in-depth evaluation of the reasons for certain problems supports the identification of improvement needs concerning documentation at the unit or department level. Identifying such problems is of high importance to support the detection, understanding and reduction of latent problems and to close gaps in the medication-related continuum of care.

Acknowledgements We thank the members of the advisory board for their valuable advice at various stages of the project: Prof. Johannes Giehl (Competence Center Quality Assurance/Management (KTQ), Medical Service of Statutory Healthcare Assurance in Germany), Prof. Ulrich Jaehde (Institute of Pharmacy, University of Bonn), Dr. Constanze Lessing (Berlin), Dr. Barbara Strohbuecker (Deutscher Pflegerat, Cologne), Prof. David Schwappach (Swiss Patient Safety Foundation, Zurich), Prof. Petra Thürmann (University Witten/Herdecke, Chair of Clinical Pharmacology; HELIOS University Clinic Wuppertal). Moreover, we would like to thank Prof. Peter Martus (Institute for Clinical Epidemiology and Applied Biometry, University Hospital of Tuebingen) for his support in the development process of MediDocQ as well as for his advice and final approval regarding the calculation of items and indices. We also thank Fatemeh Aghai, Janina Bittmann, Rebecca Geulen, Eva-Maria Müller, Jennifer Pfeiffer, Malte Ritter, Jana Rokitzki, Heiko Schulz, and Pireyanka Varatharasan for their diligent work in collecting the RCR data as well as Sigrid Emerich in supporting the coordination of data collection. In addition, we acknowledge the support of the hospital management and workers' representatives in both hospitals, the efforts of study coordinators in participating departments and units to facilitate data collection, and the patients agreeing in participating in this study. Finally, we like to thank the reviewers for BMJ Open for their helpful comments on earlier versions of this paper.

Collaborators \#During the study members of the WorkSafeMed Project Consortium were:Luntz E, Rieger MA (Project lead), Sturm H, Wagner A (Institute of Occupational and Social Medicine and Health Services Research, University Hospital of Tuebingen), Hammer A, Manser T (Institute for Patient Safety, University Hospital Bonn), Martus P (Institute for Clinical Epidemiology and Applied Biometry, University Hospital of Tuebingen), Holderried M (University Hospital Tuebingen).

Contributors AH and TM drafted the manuscript. AW and MAR contributed with valuable feedback and modifications to the text. AH and TM developed the MediDocQ instrument and were responsible for the methodological implementation of the instrument including the pilot-test. $\mathrm{AH}$ and $\mathrm{AW}$ were responsible for the data collection including the training and supervision of reviewers. AH performed the statistical analysis and received valuable feedback from the other authors. All authors read and approved the final manuscript. 
Funding The WorkSafeMed study was funded by the Federal Ministry of Education and Research (FKZ 01GY1325A and 01GY1325B). The work of the Institute of Occupational and Social Medicine and Health Services Research Tuebingen is supported by an unrestricted grant of the Employers' Association of the Metal and Electric Industry Baden-Wuerttemberg (Suedwestmetall).

Competing interests None declared.

Patient consent for publication Not required.

Provenance and peer review Not commissioned; externally peer reviewed.

Data availability statement Because of data security aspects, data from the WorkSafeMed study will not be made available in the public domain. However, data will be used by students of both project partners for their theses. Data will be stored in accordance with national and regional data security standards and might be obtained on request by contacting the first author.

Open access This is an open access article distributed in accordance with the Creative Commons Attribution Non Commercial (CC BY-NC 4.0) license, which permits others to distribute, remix, adapt, build upon this work non-commercially, and license their derivative works on different terms, provided the original work is properly cited, appropriate credit is given, any changes made indicated, and the use is non-commercial. See: http://creativecommons.org/licenses/by-nc/4.0/.

ORCID iD

Antje Hammer http://orcid.org/0000-0002-2724-9901

\section{REFERENCES}

1 Cohen MD, Hilligoss PB. The published literature on handoffs in hospitals: deficiencies identified in an extensive review. BMJ Qual Saf 2010;19:493-7.

2 Lee S-H, Phan PH, Dorman T, et al. Handoffs, safety culture, and practices: evidence from the hospital survey on patient safety culture. BMC Health Serv Res 2016;16:254.

3 Araújo MMTde, Silva MJPda, Trovo de Araújo MM, et al. Estratégias de comunicação utilizadas POR profissionais de saúde $\mathrm{Na}$ atenção pacientes sob cuidados paliativos. Rev Esc Enferm USP 2012;46:626-32.

4 Carayon P, Wood KE. Patient safety: the role of human factors and systems engineering. in: engineering the system of healthcare delivery. Amsterdam [u.a.]: IOS Press 2009:23-46.

5 Abraham J, Nguyen V, Almoosa KF, et al. Falling through the cracks: information breakdowns in critical care handoff communication. AMIA Annu Symp Proc 2011;2011:28-37.

6 Hughes RG. Nurses at the "Sharp End" of Patient Care. In: Hughes RG, ed. Patient safety and quality: an evidence-based Handbook for nurses. Rockville (MD, 2008.

7 Gutheil TG. Fundamentals of medical record documentation. Psychiatry 2004;1:26-8.

8 Mathioudakis A, Rousalova I, Gagnat AA, et al. How to keep good clinical records. Breathe 2016;12:369-73

9 Garrouste-Orgeas M, Philippart F, Bruel C, et al. Overview of medical errors and adverse events. Ann Intensive Care 2012;2:2.

10 Corrigan J, Kohn LT, Donaldson MS, eds. To err is human: Building a safer health system. Washington, D.C: National Academy Press, 2000.

11 Levinson DR. Adverse events in hospitals: national incidence among Medicare beneficiaries: department of health and human services, office of Inspector General. Report OEl-06-09-00090.

12 Weiss AJ, Elixhauser A, Bae J, et al. Origin of adverse drug events in U.S. hospitals, 2013. Available: www.hcup-us.ahrq.gov/reports/ statbriefs/sb158.jsp [Accessed 22 Sep 2017].

13 Huckels-Baumgart S, Manser T. Identifying medication error chains from critical incident reports: a new analytic approach. J Clin Pharmacol 2014;54:1188-97.

14 Hartel MJ, Staub LP, Röder C, et al. High incidence of medication documentation errors in a Swiss university hospital due to the handwritten prescription process. BMC Health Serv Res 2011;11.

15 Routledge PA. A national in-patient prescription chart: the experience in Wales 2004-2012. Br J Clin Pharmacol 2012;74:561-5.

16 Coombes ID, Reid C, McDougall D, et al. Pilot of a national inpatient medication chart in Australia: improving prescribing safety and enabling prescribing training. Br J Clin Pharmacol 2011;72:338-49.

17 Australian Commission on Safety and Quality in Health Care. Electronic medication management systems: a guide to safe implementation. $3^{\text {rd }}$ edn. Sydney, 2017.
18 Vassar M, Holzmann M. The retrospective chart review: important methodological considerations. J Educ Eval Health Prof 2013;10:12.

19 Thomas EJ, Petersen LA. Measuring errors and adverse events in health care. J Gen Intern Med 2003;18:61-7.10.1046/j.15251497.2003.20147.x

20 Rozich JD, Haraden CR, Resar RK. Adverse drug event trigger tool: a practical methodology for measuring medication related harm. Qual Saf Health Care 2003;12:194-200.

21 Preen DB, Holman C. D'Arcy J., Lawrence DM, et al. Hospital chart review provided more accurate comorbidity information than data from a general practitioner survey or an administrative database. $J$ Clin Epidemiol 2004;57:1295-304.

22 Freres M, Walter C. Behandlungsfehler im Krankenhaus: Offenlegen - entschuldigen - entschädigen. Dtsch Ärtzeb/ 2013;110:A-1848 / B-1632 / C-1602.

23 Groves PS. The relationship between safety culture and patient outcomes: results from pilot meta-analyses. West J Nurs Res 2013;36:66-83.

24 European Commission. Key findings and recommendations on: reporting and learning systems for patient safety incidents across Europe: European Commission 2014.

25 Laatikainen O, Miettunen J, Sneck S, et al. The prevalence of medication-related adverse events in inpatients - a systematic review and meta-analysis. Euro J Clin Pharmacol 2017:1-11.

26 Wilmer A, Louie K, Dodek P, et al. Incidence of medication errors and adverse drug events in the ICU: a systematic review. BMJ Qual Saf 2010;19:e7

27 Keers RN, Williams SD, Cooke J, et al. Causes of medication administration errors in hospitals: a systematic review of quantitative and qualitative evidence. Drug Saf 2013;36:1045-67.

28 Peusschers E, Twine J, Wheeler A, et al. Documentation of medication changes in inpatient clinical notes: an audit to support quality improvement. Australas Psychiatry 2015;23:142-6.

29 Armutlu M, Foley M-L, Surette J, et al. Survey of nursing perceptions of medication administration practices, perceived sources of errors and reporting behaviours. Hcq 2008;11:58-65.

30 Barker KN, Flynn EA, Pepper GA, et al. Medication errors observed in 36 health care facilities. Arch Intern Med 2002;162:1897-903.

31 Johari H, Shamsuddin F, Idris N, et al. Medication errors among nurses in government Hospital 2013.

32 Callen J, Mclntosh J, Li J. Accuracy of medication documentation in hospital discharge summaries: a retrospective analysis of medication transcription errors in manual and electronic discharge summaries. Int J Med Inform 2010;79:58-64.

33 Callen JL, Alderton M, Mclntosh J. Evaluation of electronic discharge summaries: a comparison of documentation in electronic and handwritten discharge summaries. Int J Med Inform 2008;77:613-20.

34 Adeleke IT, Adekanye AO, Onawola KA, et al. Data quality assessment in healthcare: a 365-day chart review of inpatients' health records at a Nigerian tertiary hospital. J Am Med Inform Assoc 2012;19:1039-42.

35 Lucado J, Paez K, Elixhauser A. Medication-Related Adverse Outcomes in U.S. Hospitals and Emergency Departments, 2008: Statistical Brief \#109. In: Healthcare cost and utilization project (HCUP) statistical Briefs. Rockville, MD, 2006.

36 Zegers M, de Bruijne MC, Spreeuwenberg P, et al. Quality of patient record keeping: an indicator of the quality of care? BMJ Qual Saf 2011;20:314-8.

37 Wagner A, Rieger MA, Manser T, et al. Healthcare professionals perspectives on working conditions, leadership, and safety climate: a cross-sectional study. BMC Health Serv Res 2019;19:53.

38 Cousein E, Mareville J, Lerooy A, et al. Effect of automated drug distribution systems on medication error rates in a short-stay geriatric unit. J Eval Clin Pract 2014;20:678-84.

39 Baron RJ. Quality improvement with an electronic health record: achievable, but not automatic. Ann Intern Med 2007;147:549-52.

40 Evans RS. Electronic health records: then, now, and in the future. Yearb Med Inform 2016;25:S48-61.

41 Hunt KV, Harding AM, Taylor SE, et al. Evaluation of medication dose omissions amongst inpatients in a hospital using an electronic medication management system. J Eval Clin Pract 2018;24:688-94.

42 Sträßner HR. Rechtliche Aspekte Der Pflegedokumentation. CNEF 2010;4:2-8.

43 Cain C, Haque S. Patient safety and quality: an evidence-based Handbook for nurses: organizational workflow and its impact on work quality. Rockville, MD, 2008.

44 First C. Medical record documentation standards. $5129^{\text {th }}$ edn. Maryland, 2016.

45 Stevens S, Pickering D. Keeping good nursing records: a guide. Community Eye Health 2010;23:44-5. 